\title{
Rare congenital fistula connection between right subclavian artery and superior vena cava presenting in neonate with congestive cardiac failure
}

\begin{abstract}
This case report describes a congestive cardiac failure in neonate secondary to rare congenital arteriovenous fistula between the right subclavian artery and superior vena cava. The abnormality was initially discovered by echocardiography and then confirmed by CT angiography. The neonate got successful surgical repair and uneventful post surgical period. Intra-thoracic congenital arteriovenous malformations are very rare and presenting early with cardiac failure, which is challenging for diagnosis and treatment. However early identification and treatment is the most influencing factor in mortality and morbidity of these cases.
\end{abstract}

Keywords: arteriovenous malformation, subclavian artery, fistula, congestive cardiac failure
Volume 8 Issue 3 - 2018

\author{
Awadh Salem Al-Majrafi,' Naela Khalifa Al \\ Kalbani, ${ }^{2}$ Farida Ambusaidi ${ }^{3}$ \\ 'Senior specialist pediatric, Pediatric Department Ibri Hospital, \\ Sultanate of Oman \\ ${ }^{2}$ Medical Officer, Radiology Department lbri Hospital, Sultanate \\ of Oman \\ ${ }^{3}$ Pediatric radiologist Specialist, Radiology Department Ibri \\ Hospital, Sultanate of Oman
}

\begin{abstract}
Correspondence: Farida Ambusaidi, Pediatric radiologist Specialist, Radiology Department Ibri Hospital, Sultanate of Oman,Tel 00968997938I0,Email farida.ambusaidi@gmail.com
\end{abstract}

Received: March 28, 2018| Published: June 28, 2018

\section{Introduction}

Intra-thoracic arteriovenous malformations are uncommon. As the most reported localizations of AVM are the head (vein of Galen malformation), the abdomen (infantile hepatic hemangioma), the neck and extremities. ${ }^{1}$ Arterio venous malformation divided into congenital and acquired. The congenital forms are even more uncommon and patients present with variable symptoms that make the diagnosis more challenging. ${ }^{2,3}$ A congenital Aortocaval fistula from subclavian artery to the superior vena cava (SVC) may represent a subclass of this condition.

\section{Case report}

Our patient is full term to primigravida mother born by spontaneous vaginal delivery with good Apgar score and birth weight of 3.48 $\mathrm{kg}$. Baby required no resuscitation. No risk factors for sepsis. Baby developed tachypnea at 2 hours of life, so shifted to Special care Baby Unit (SCBU). Examination revealed bruit over right subclavian area.

Echocardiography was done on day one of life and showed:

a. Situs solitus, levocardia.

b. The SVC was dilated and had high pulsatile flow.

c. The RA and right ventricle (RV) were moderately dilated.

d. Mildly Poor ventricular function, ejection fraction (EF) $51 \%$.

e. Mean pulmonary artery (MPA) was dilated $(9.2 \mathrm{~mm})$ and branches are normal.

f. Pulmonary veins all present with normal drainage to left atrium (LA).

g. There was a $3 \mathrm{~mm}$ patent ductus arteriosus (PDA) with mainly Rt to LT flow.

h. Arch was left and the first branch was dilated. There was significant diastolic runoff in the transverse and descending aorta, no narrowing or sign of CoA.

Impression: Extra cardiac arteriovenous shunt, Right subclavian artery (SCA) to SVC

Baby started on anti failure medications. The baby deteriorated on day 2 of life, so incubated. Echo repeated with same findings plus newly developed pulmonary hypertension (Figure 1) (Figure 2).

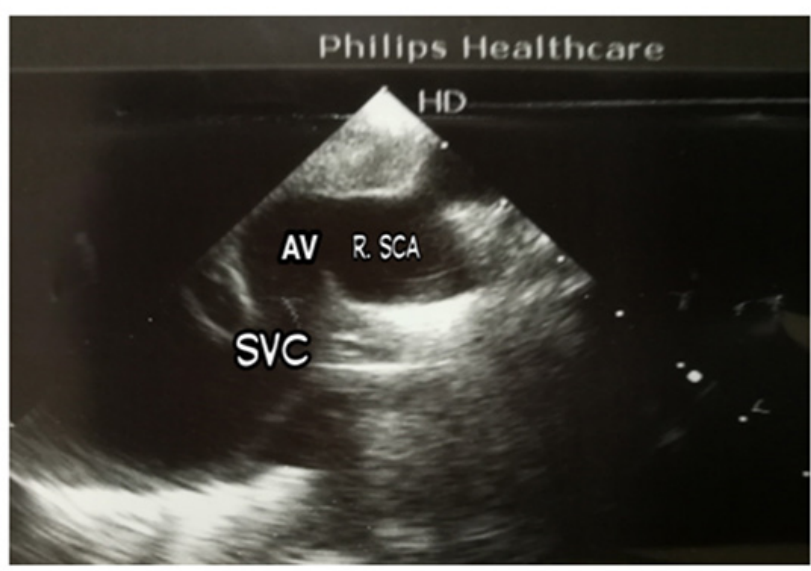

Figure 1 Short axis view Echocardiography for the AV connection between the dilated SCA to Dilated SVC.

Baby started on anti failure medications. The baby deteriorated on day 2 of life, so incubated. Echo repeated with same findings plus newly developed pulmonary hypertension.

\section{*CT Angiography showed:}

a. Anomalous vessels connecting proximal right subclavian artery with superior vena cava, representing arteriovenous fistula.

b. Cardiomegaly and right sided heart strains likely related to arteriovenous shunting. 

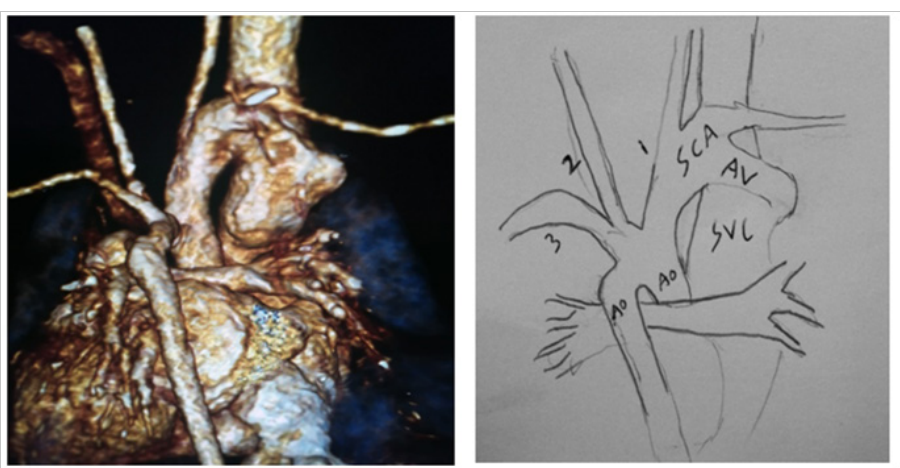

Figure 2A, Figure 2B 3D CT angio for the great arteries and the $A V$ malformation, (B) Diagram illustration of the AV malformation in figure (A). *USG head was done and showed no obvious vein of Galen malformation. The baby was transferred to the national cardiac center where he got repair of right subclavian artery to superior vena cava arterio-venous fistula (ligation and division) and ligation of patent ductus arteriosus.

\section{Discussion}

Aortocaval fistulas are rare cause of left-to-right-shunt resulting in neonatal congestive heart failure. The vessels initially develop from the mesenchyma in an undifferentiated form till give fully developed veins and arteries. So, they develop from same primordial network of vessels. Congenital arteriovenous fistulas may be the result, if these early connections of both arteries and veins persist. Hereby congenital AVM is a mal-development of blood vessels, with preservation of one or more primitive direct communications between arterial and venous channels. ${ }^{4,5}$ Histological, the fistula connection shows hyperplasia of elastic fibers of the arterialized vein, ranging from 41 to $82 \%{ }^{6,7}$ There are few cases in literature similar to our case, right subclavian artery to SVC fistula reported in 1977 by Arkell and Lawson ${ }^{8}$, in 2011 case by Balakrishnan et al., ${ }^{9}$ and another case by Awasthy et al. ${ }^{10}$. Three case reported by Gutierrez et al., ${ }^{2}$ two of them showed a fistula between left subclavian artery-to-innominate vein and draining into markedly dilated superior vena cava. Sapire et al. ${ }^{11}$ In 1983 reported a case of arterio-venous fistula between the subclavian artery and in nominate vein presented with heart failure in neonatal period. ${ }^{11}$ In 2000 Dogan et al., ${ }^{12} \&$ Tatum et al., ${ }^{13}$ in 2006 reported congestive heart failure due to subclavian artery to subclavian vein fistula. In 2016, Sami Jabari \& Robert Cesnjevar ${ }^{14}$ reported a case of fistulatous connection between Brachiocephalic trunk and superior vena cava. The echo cardio graphy is simple fast screen tools for cardiac abnormality and first alert to such entity. The confirmation by using 64 slice CT scan was hand-to-hand a great support that confirm the diagnosis which facilitate the transfer of patient to the appropriate institution to get the proper treatment.

\section{Conclusion}

The early presentation of heart failure in neonatal period necessitate extensive work up excluding all arteriovenous malformation in heart, head, chest and abdomen. This case highlights a rare congenital arteriovenous fistula connection between subclavian artery and superior vena cava. Echocardiography is important non invasive tool in diagnosing and raising suspicion of congenital Aortocaval arteriovenous fistula. CT angiography was complementary tool to diagnose the malformation.

\section{Funding details}

No funding agency.

\section{Acknowledgements}

None.

\section{Conflict of interests}

The author declares there is no conflict of interest.

\section{References}

1. Knudson RP, Alden ER. Symptomatic arteriovenous malformation in infants less than 6 months of age. Pediatrics. 1979;64(2):238-241.

2. Gutierrez FR, Monaco MP, Hartmann Jr AF, et al. Congenital arteriovenous malformations between brachiocephalic arteries and systemic veins. Chest. 1987;92(5):897-899.

3. Jabari S, Hartmann A, Cesnjevar R. A rare case of congenital thoracic arteriovenous fistula between the brachiocephalic truncus and the superior vena cava resulting in heart failure. Journal of Pediatric Surgery Case Reports. 2016;15:50-52.

4. Moore KL, Viedhya T, Persaud N. The developing human. $7^{\text {th }}$ ed. Saunders; 2003.

5. Hinrichsen KV. Human embryology: textbook and atlas of prenatal development of man. Berlin, Heidelberg; Springer-Verlag; 1990.

6. Takami T, Ohata K, Nishio A, et al. Histological characteristics of arterialized medullary vein in spinal dural arteriovenous fistulas related with clinical findings: report of five cases. Neurology India. 2006;54(2):202-204.

7. Massoud TF, Vinters HV, Chao KH, et al. Histopathologic characteristics of a chronic arteriovenous malformation in a swine model: preliminary study. AJNR Am J Neuroradiol. 2000;21(7):1268-1276.

8. Arkell DG, Lawson LJ. Subclavian-superior vena caval arteriovenous fistula. Vascular and Endovascular Surgery. 1977;11(2):94-98.

9. Balakrishnan PL, Guleserian KJ, Schuster J, et al. Congenital arteriovenous malformation between the subclavian artery and superior vena cava presenting in neonatal heart failure. Pediatr Cardiol 2011;32(80:1204-1206.

10. Awasthy N, Tomar M, Radhakrishnan S, et al. Nonsurgical management of a congenital aortocaval fistula from right subclavian artery to superior vena cava along with SVC obstruction. Pediatr Cardiol. 2011;32(2):227229.

11. Sapire DW, Lobe TE, Swischuk LE, et al. Subclavian artery-toinnominate-vein fistula presenting with congestive failure in a newborn infant. Pediatr Cardiol. 1983;4(2):155-157.

12. R Dogan, M Yılmaz, S Özkutlu, et al. Congenital Subclavian Artery to Subclavian Vein Fistula Presenting with Congestive Heart Failure in an Infant. Pediatric Cardiology. 2000;21(3):269-270.

13. Tatum GH, Newman B, Ralphe JC. Neonatal congestive heart failure due to a subclavian artery to subclavian vein fistula diagnosed by noninvasive procedures. Congenit Heart Dis. 2006;1(3):120-123.

14. Sami Jabari, Robert Cesnjevar. A rare case of congenital thoracic arteriovenous fistula between brachiocephalic truncus and the superior vena cava resulting in heart failure. Journal of Pediatric Surgery Case Reports. 2016;15:50-52. 